\title{
Observer based Dynamic Adaptive Cooling System for Power modules
}

\author{
Xiang Wang, Alberto Castellazzi, Pericle Zanchetta \\ The Power Electronics, Machines and Control (PEMC) Research Group, Electrical \\ Systems and Optics Research Division, Department of Electrical and Electronic \\ Engineering, University of Nottingham, Nottingham, NG7 2RD, United Kingdom (UK).
}

\begin{abstract}
This paper presents an advanced dynamic cooling strategy for multi-layer structured power electronic modules. A observer based feedback controller is proposed to reduce a power device or module's thermal cycle amplitude during operation, with the aim of improving reliability and lifetime. The full-state observer design is based on a developed Cauer type thermal model. The observer enables estimation and control of the temperature at reliability critical locations only measuring one accessible location. This makes the method particularly powerful and suitable for application in power systems. The designed strategy is confirmed experimentally. Although the experiment is developed for a specific application scenario, the proposed strategy is of general validity.
\end{abstract}

Keywords: Cooling, Reliability, Temperature Control, Thermal Stress, Thermal Cycle, Lifetime

Email addresses: eexxw13@nottingham.ac.uk (Xiang Wang), alberto.castellazzi@nottingham.ac.uk (Alberto Castellazzi), pericle.zanchetta@nottingham.ac.uk (Pericle Zanchetta) 


\section{Introduction}

In power electronics, the failure mechanisms generally can be grouped by random and wear-out failures $[1,2,3]$. Wear-out mechanism failures make up the majority of failures in power electronic modules [4]. In wear-out mechanism, thermo-mechanical stress plays a very important role in affecting power electronic devices/modules reliability [1,5], such as the fractures propagation and degradations in solder layers [6], wire-bond lift-off [7] and emitter metallization [8]. The failure mechanisms are influenced by both environmental and load conditions $[9,10]$. To address this issue, research has addressed different aspects, for example, new semiconductor and materials technologies [11, 12], package architecture [13], interconnection [14], control of power electronic modules [15] and advanced cooling technologies [5, 16].

Fig. 1 describes a summary of the results of extensive reliability tests on IGBT power modules [17]. These results clearly indicated that, over the considered temperature range, a power module operational lifetime depends mainly on two parameters: 1 ) the amplitude of the thermal cycles, $\Delta \mathrm{T}$, that the module experiences; 2) the average operational temperature, Tm. Fig. 1 clearly shows that if $\Delta \mathrm{T}$ is reduced by even the same amount that $\mathrm{Tm}$ is increased, a much higher number of cycles to failure can be achieved. For instance, moving from point 1 to point 2 , as $\Delta \mathrm{T}$ is fixed at $50 \mathrm{~K}$, increasing Tm by $20 \mathrm{~K}$ from $80^{\circ} \mathrm{C}(353.15 \mathrm{~K})$ to $100^{\circ} \mathrm{C}(373.15 \mathrm{~K})$, the cycles to failure will be reduced $3 \times 10^{5}$ cycles from point $1\left(5 \times 10^{5}\right.$ cycles $)$ to point $2\left(2 \times 10^{5}\right.$ cycles). However, moving from point 2 to point 3, keeping the same Tm, a reduction of $20 \mathrm{~K}$ in $\Delta \mathrm{T}$ increases the number of cycles to failure to $2 \times 10^{6}$, that is, even better than the starting point 1 . In other words, quantitatively 
$\Delta \mathrm{T}$ has a much more significant effect on the reliability of power modules than Tm.

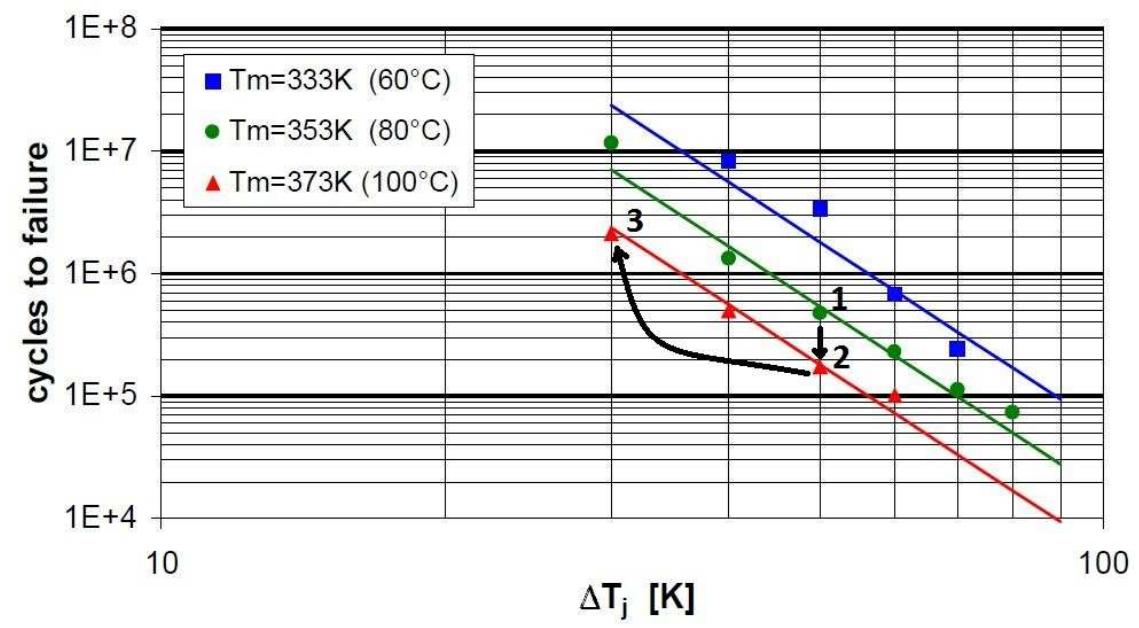

Figure 1: Reliability of power modules as a function of thermal cycle amplitude for different values of average temperature [17]

Presently, typical power device thermal management only aims at ensuring that the maximum operating temperature is kept below a safety critical value at full-load or worst-case conditions and the cooling device is based on fixed designed parameters. In view of the close considerations, from a reliability point of view, this is clearly not optimum. Some temperature regulated thermal management strategies have been proposed with consideration of maintaining device operation temperature variation as small as possible $[18,19,20]$. A temperature control system is presented in [18], where the device under test (DUT) is sandwiched with a heat sink and heater. By electrically controlling the heater power, heat flow to/from the electronic device is quickly adjusted; and that in turn regulates the device temperature. This strategy is actually a heating strategy instead of a cooling strategy and it 
costs extra power for heating the device to a certain temperature value. The patent in [19] demonstrates a temperature controlled cooling method. In this method, the DUT is cooled by mechanically swing the cooling fluid direction (e.g. the cooling fan facing direction) towards the heat dissipation element to regulate the temperature to a target value. However, this method requires several parallel mounted cooling fans and each fan needs a motor for swing functions, which increases the complexity of the cooling system and limits its thermal response time. In [20], a method to control the fan speed used in cooling integrated circuits is presented. In this method, a thermal diode is used to monitor device temperature, and the fan speed is adjusted by looking up a pre-defined temperature-speed table. There are two main shortages for this method: 1) a temperature sensor must be mounted inside the device; 2) controlling cooling fan by look-up table is an open loop control thus it is sensitive to system variations and easy to have temperature errors. Therefore, considering the reliability and temperature control issues, an observer-based adaptive cooling strategy with multi-variable feedback control technique is proposed here.

As shown in Fig. 2, the temperature with constant cooling power will vary as load changes. In order to decrease $\Delta \mathrm{T}$, the cooling power can be adjusted according to the load variations and this can be achieved simply by reducing the cooling power. 


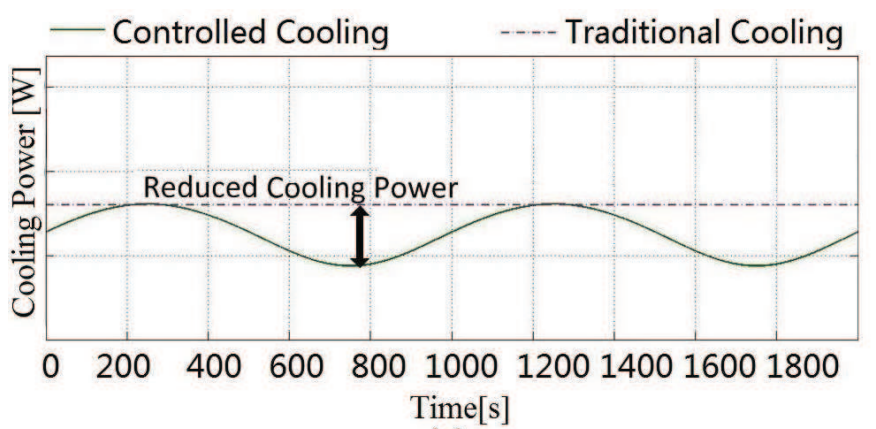

(a)

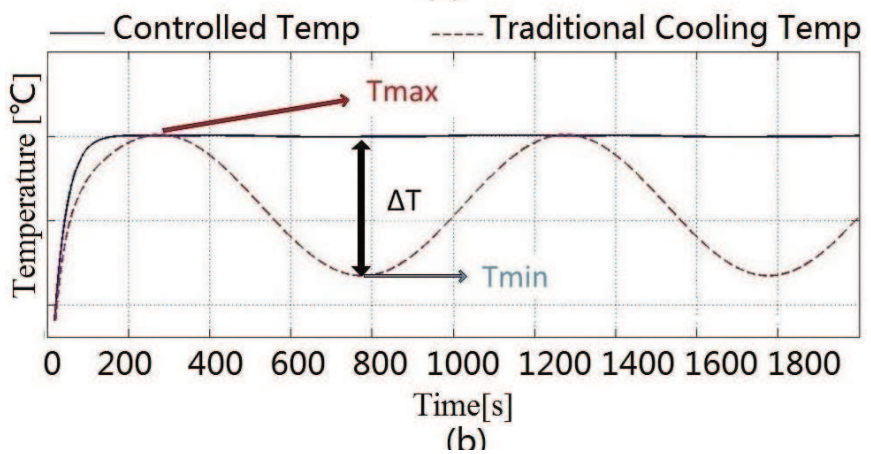

Figure 2: Change in thermal cycling with traditional cooling approach and with the proposed cooling strategy: (a) Cooling power (b) Temperature

This paper presents an advanced dynamic cooling strategy for multi-layer structured power electronic modules. An observer based feedback controller is proposed to reduce a power device or module's thermal cycle amplitude during operation, with the aim of improving reliability and lifetime. The proposed methodology is schematically illustrated in Fig. 3:

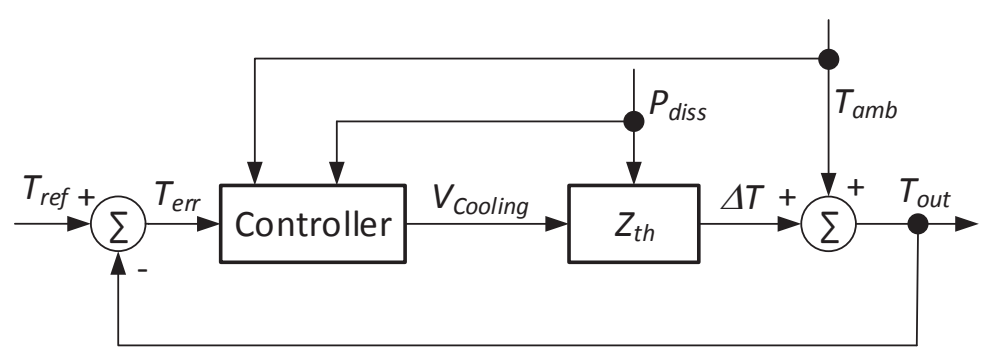

Figure 3: Block diagram for the control design 
the temperature at a reliability critical location of the power assembly is controlled against variations in the actual load and power losses $P_{\text {diss }}$ (i.e., power dissipation) and boundary condition $T_{a m b}$ (i.e., ambient temperature). The feedback control loop monitors the temperature of the desired location $T_{\text {out }}$ and intervenes on the cooling parameter $V_{\text {cooling }}$ to eliminate temperature errors $T_{e r r}$ to control the temperature output and decrease temperature variations. The control parameter $V_{\text {cooling }}$ is the controller output signal used to control the cooling devices. It can be the bias voltage applied on the fan for a forced air convection cooling, or the voltage on the pump in a liquid cooling system. By controlling the cooling device, the thermal impedance of the system, $Z_{t h}$, is adjusted to meet the temperature regulation. A observer based feedback controller is proposed to reduce a power device or modules thermal cycle amplitude during operation, with the aim of improving reliability and lifetime. The full-state observer design is based on a developed Cauer type thermal model. To ensure the accuracy of the developed model, FEA (Finite Element Analysis) method is applied to deriving a Cauer type thermal network where the observer is modelled on. The observer enables estimation and control of the temperature at reliability critical locations only measuring one accessible location. This makes the method particularly powerful and suitable for application in power systems. The designed strategy is confirmed experimentally. Although the case-study experiment is developed for a specific application scenario, the proposed strategy is of general validity. 


\section{Temperature Estimation}

A common way to estimate junction temperature is building a real-time thermal model and match the model to experiment data to get a reference look-up table for temperature estimation [21]. This requires high accuracy physical parameters, proper initial conditions to ensure the precision of modelling, high initial efforts to build up the look-up table and basically only for junction temperature estimation. Here, the proposed temperature full-order observer is a system that provides an estimation of the internal states (temperatures) of a given real system (power module). The state-space thermal model is based on module's physical structure and material properties, thus with knowing the system's inputs $P_{\text {diss }}$ (i.e., power dissipation), $T_{a m b}$ (i.e., ambient temperature) and output (i.e., the temperature at any certain layer location inside the module), the observer will be able to calculate the junction temperature and temperatures at other layers inside the module. This enables that many modules have inbuilt temperature sensors [22] can be used with the proposed observing method. The system states are necessary to solve many control theory problems so that the state observer can be used in investigating the critical temperature location (e.g. junction temperature or solder layer temperature) in active temperature control applications. Because the temperatures are treated as internal states in the observer, the temperature information at all physical layers can be achieved at same time.

For validation purposes a simple test assembly was produced as shown in Fig. 4, and the validation process and results are discussed in detail in early works [23]. This consists of an IGBT to be used as the heating element and a diode to be used as temperature sensor; the top surface of the IGBT (source 
terminal) was contacted with hollow copper bumps, into which a thermocouple (here, a K-type thermocouple was used) was inserted to provide a second temperature measurement point, much closer to the actual heat source (i.e., the IGBT chip). The IGBT is driven in the on-state with a constant gateemitter voltage; a variable DC voltage source is connected to collector and emitter to generate variable power dissipation; the diode, previously duly calibrated, is biased with constant current $(500 \mathrm{~mA})$ to monitor variations of its forward voltage drop with temperature.

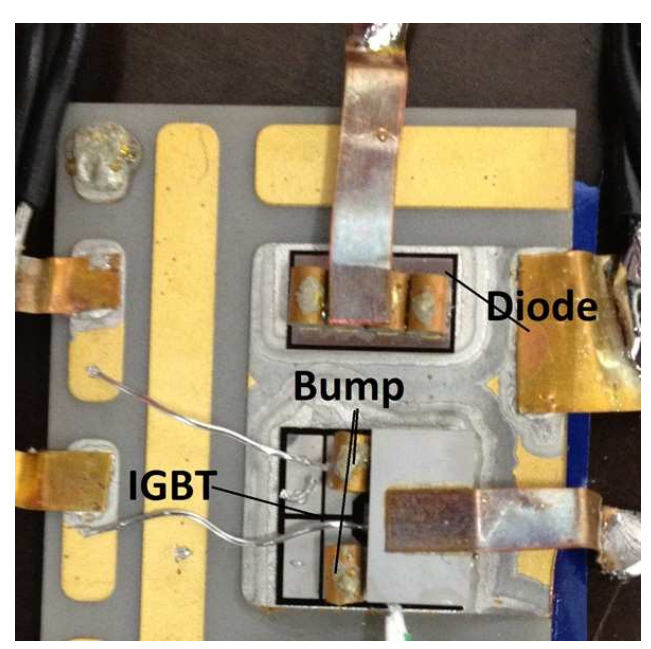

Figure 4: Tested IGBT assembly

The Cauer type thermal network is built because it can represent a real physical heat-flow path. To ensure the accuracy of the thermal model, the thermal resistance and thermal capacitance values in Cauer network are derived from FEA simulation results [24]. The test model is built in 3-D with FEA software, Abaqus. In FEA simulation, assuming a $100 \mathrm{~W}$ power is dissipated and the top $1 / 3$ part of the silicon layer of IGBT is set to the heat 
source. Adiabatic boundary conditions are applied to the top and lateral surfaces and convective boundary conditions are applied to the bottom surface only (heat-sink). As mentioned above, for most cooling conditions and assembly structures, heat conduction can be assumed to take place essentially in the vertical direction, from the chips towards the heat-sink, enabling for an essentially 1-D treatment of the problem. This allows derivation of the associated thermal resistance $R_{i}$, and thermal capacitance, $C_{i}$, of each layer. Therefore, the temperature measuring locations are set to the elements beneath the centre of the IGBT gate chip, and in the center of each material layer. To verify the extracted parameters, a Cauer type thermal model is simulated in Simulink with the same load parameters in Abaqus, and the simulated temperature results are compared with the original FEA results.

Based on the derived Cauer network, the thermal model can be formalised in the framework of state-space description. Based on this state-space model, a full-order state observer can be built in the form of Fig. 5 [23]:

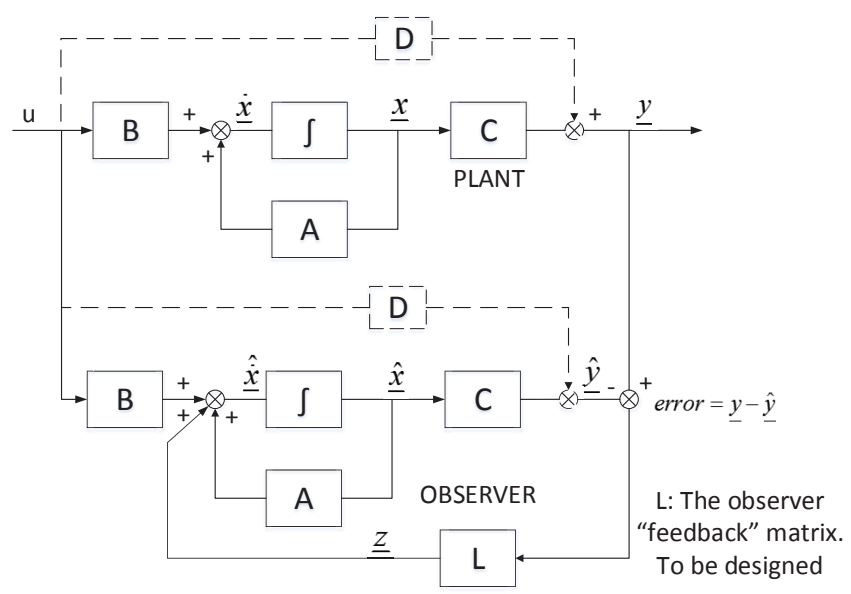

Figure 5: Full-state observer scheme 


\section{Temperature Control System Design}

Based on the proposed thermal modelling method and the temperature observer, the adaptive cooling system controller can be designed. Usually, the equations of the thermal model involving the controllable thermal impedance $Z_{t h}$ (or using thermal resistance $R_{\text {cooling }}$ for simplicity) and the cooling parameter $V_{\text {cooling }}$ are non-linear. However, modern control system design is mainly based on linear system design: analysis methods are much easier for linear models than for non-linear models. Therefore, the developed thermal model must be linearised so to allow control methods to be applied and achieve the expected thermal performance.

The thermal system used in the experiment is modelled based on an Cauer-type electrical equivalent circuit for the assembly including the cooling device. In the circuit structure shown in Fig. 6, each node of the network corresponds to an actual physical location of the assembly: voltage corresponds to temperature and current to heat-flow.

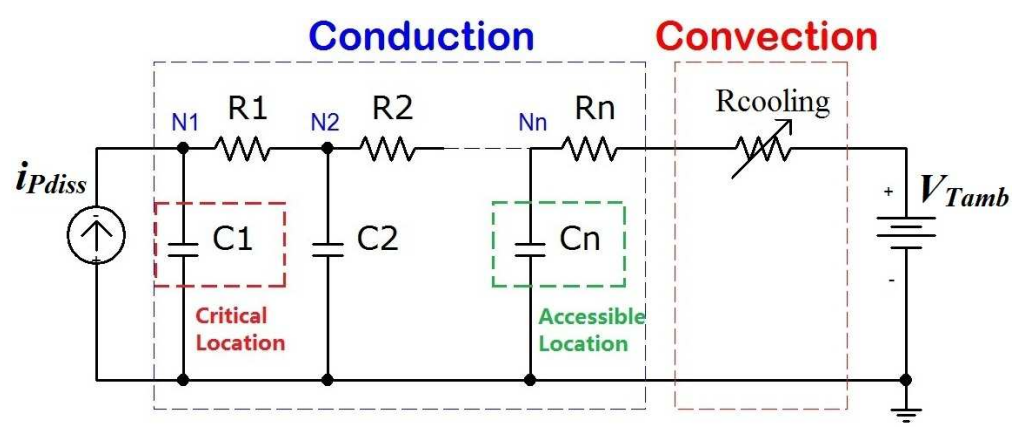

Figure 6: Thermal-Electrical equivalent circuit of a typical assembly

Here, the convective boundary conditions are emulated by means of a variable thermal resistance, $R_{\text {cooling, }}$, which corresponds to the heat-sink as 
well as the convection part. The action of the convection cooling is modelled by Equation (1), where the cooler's thermal resistance, $R_{\text {cooling }}$, is a function of control parameters (e.g., the bias voltage of a fan or that of a liquid cooler's pump). Here, $R_{\text {cooling }}$ becomes the control variable and it will be adjusted during the operation.

$$
\Phi=\frac{T_{n}-T_{a m b}}{R_{n}+R_{\text {cooling }}}
$$

Based on the derived equivalent thermal model, the state-space model used for the temperature controller design can be built, and the detailed procedure is described in $[25,26]$. Note that due to the non-linearity of $R_{\text {cooling }}$ and $V_{\text {cooling }}$, the state-space system must be linearised before designing controller with it.

In the state-space system, among the inputs, only $V_{\text {cooling }}$ is the controllable signal, and both $P_{\text {diss }}$ and $T_{a m b}$ are decided by the loading and the external environment so that they can be treated as system disturbances. To control the output temperature at the desired value accurately, the system output temperature must present zero steady-state error to a step command. However, using the multivariable state feedback control on its own, any change in the model parameters will cause the error to be non-zero. Therefore, integral control is introduced. Then, the open-loop system eigenvalues (poles) of the augmented system can be calculated and the closed-loop poles can be placed according to the desired dynamic response performance by the feedback law. A detailed feedback controller design and validation on a preliminary assembly was published earlier in [25]. 


\section{Reliability Testing: Proof of Concept Demonstration}

A photograph of the test vehicle is shown in Fig. 8: it is a commercial IGBT module mounted on a fanned heat-sink.

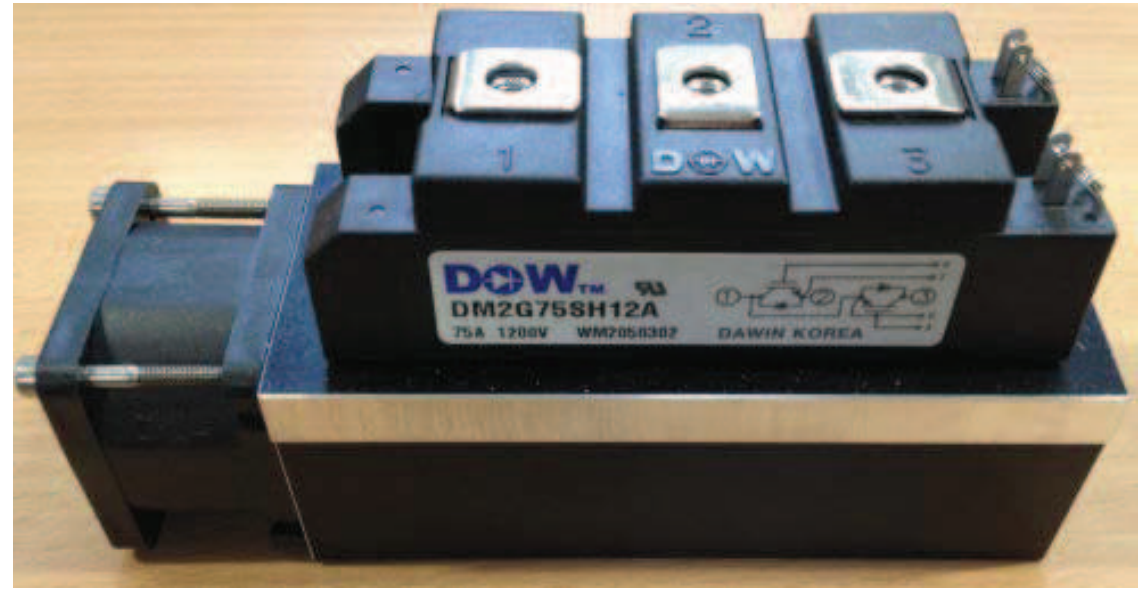

Figure 7: Tested IGBT module on heat-sink

It was subjected to 3000 temperature and power cycles derived from a realistic mission profile for wind applications, shown in Fig. 8. The temperature at baseplate was measured during operation, a certain part of two cycles is shown in Fig. 9. With the collected baseplate temperature and the proposed observer, the reference temperature at the solder layer beneath the substrate is set to $80{ }^{\circ} \mathrm{C}$, which is the peak solder layer temperature with fixed cooling. However, during the steady state, the thermal chamber used in this experiment shifts its temperature somewhat so that the temperature with fixed cooling shifts as well. But, the controlled one can still reach its reference temperature by adjusting its cooling power, therefore a peak temperature difference $T_{\text {diff }}$ is generated as shown in Fig. 9. From the measured baseplate temperature profile we can clearly see that although the controller 
cannot keep temperature constant during the whole cycling due to the harsh ambient temperature change, it still can reduce almost $45^{\circ} \mathrm{C}$ in $\Delta T$ with only increasing $20^{\circ} \mathrm{C}$ in its average temperature. This $25^{\circ} \mathrm{C}$ temperature difference can improve the device reliability a lot.

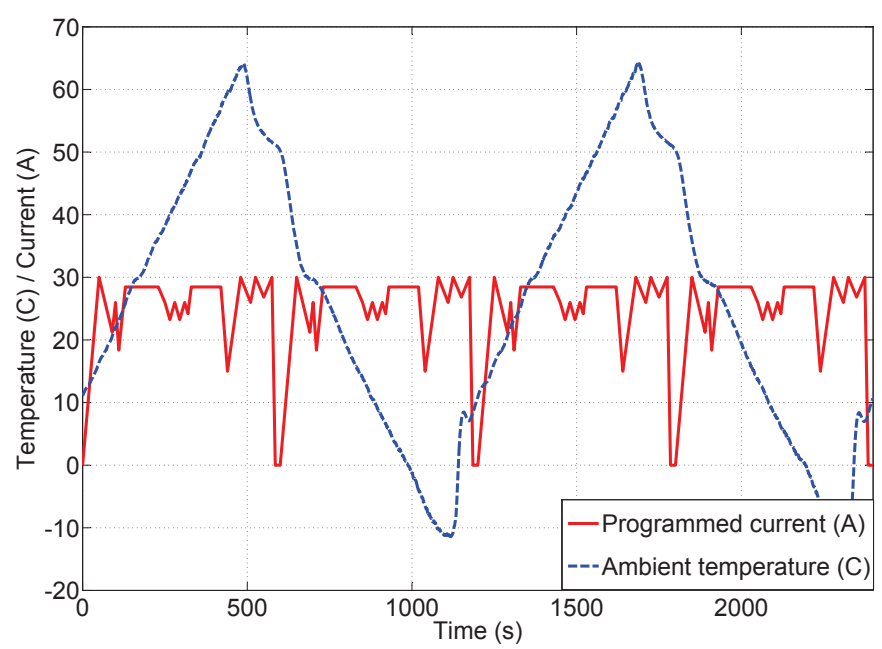

Figure 8: Long-term degradation test conditions for active and passive thermal cycling

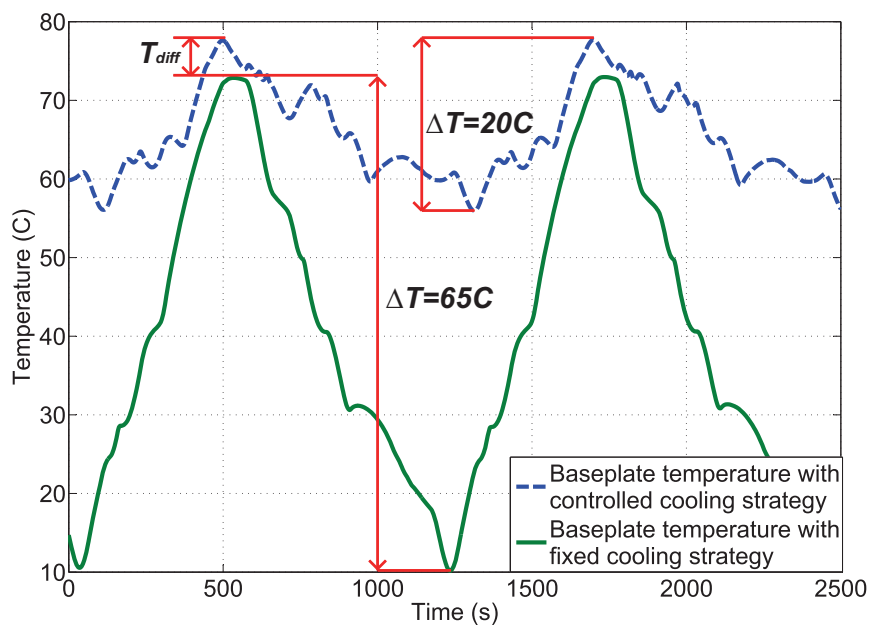

Figure 9: Measured temperature variation at the baseplate for the test conditions of Fig. 8, with and without the proposed regulated cooling strategy, respectively 
The proofs of reliability and lifetime improvement are demonstrated by checking the tested IGBT module solder layers, which are scanned before and after the test for both of the adaptive cooling and fixed cooling solutions. The SAM (Scanning Acoustic Microscope) scanned pictures are shown in Fig. 10 and Fig. 11.

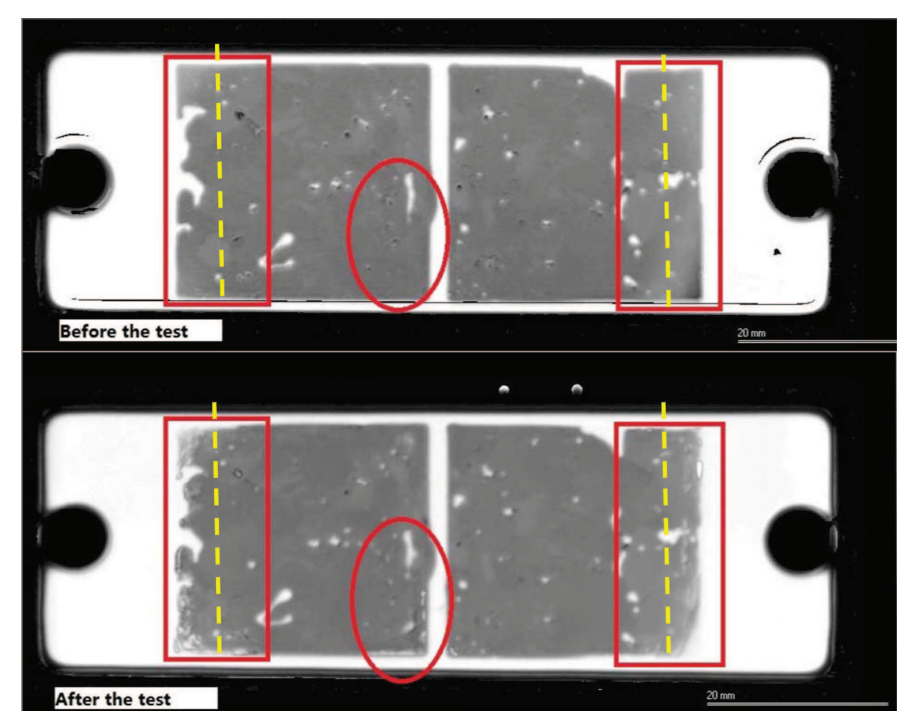

Figure 10: Fixed cooling: Tested IGBT module baseplate solder layer SAM scan results after 3000 temperature cycles.

Fig. 10 shows the tested IGBT module baseplate solder layer SAM scan results with fixed traditional cooling strategy after 3000 temperature cycles: the comparison of results before and after the test shows that degradation and solder delamination happened around the solder layer corner first under the test (indicated in the red areas). 


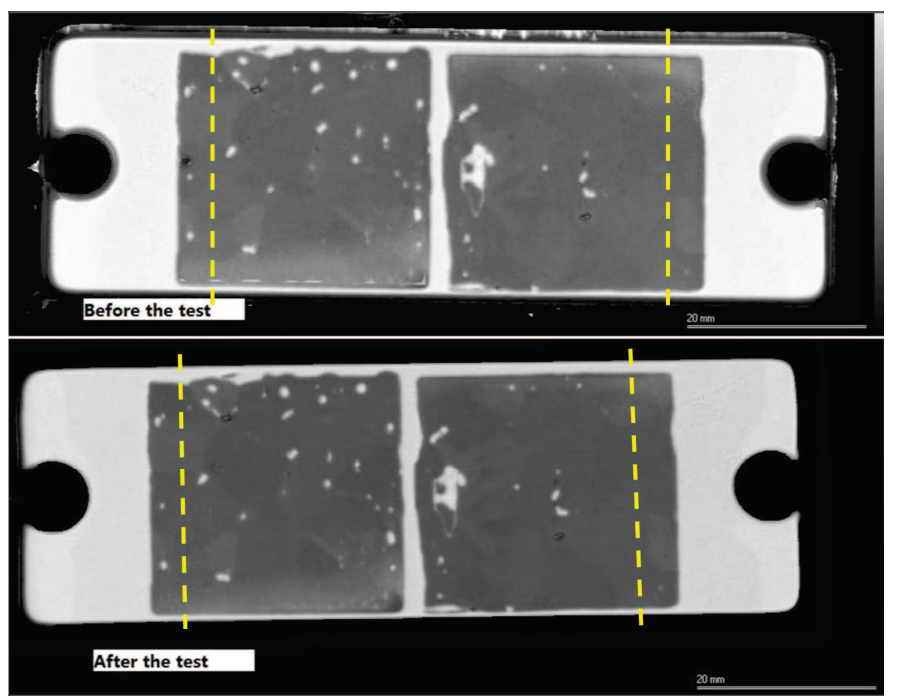

Figure 11: Adaptive controlled cooling: Tested IGBT module baseplate solder layer SAM scan results after 3000 temperature cycles.

Fig. 11 shows the tested solder layer SAM scan results with the proposed adaptive cooling strategy after 3000 temperature cycles: the comparison of results before and after the test shows that by regulating the temperature variation amplitude during cycling, there is no obvious degradation. These results prove that the proposed adaptive cooling improves solder layer reliability, as indicated in the red area along the edge side.

To have a more detailed observation of the tested device, cross-section examination of the devices near solder layer edge (the location as the yellow dashed lines in Fig. 10) are scanned by SEM (Scanning Electron Microscope), as shown in Fig. 12 and Fig. 13. 


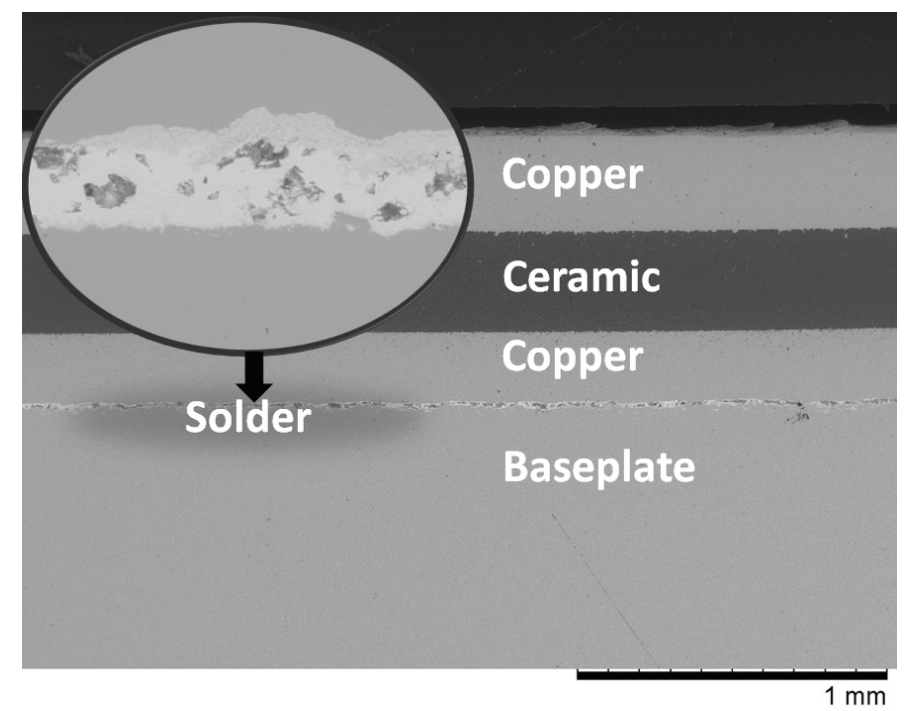

Figure 12: SEM cross-section scanning for fixed cooling solution

In the case of traditional fixed cooling, shown in Fig. 12, the baseplate solder layer has cracks and voids generated by the cycling test and the solder layer becomes thinner along its edge due to the warpage of the device during thermal cycling [27]. 


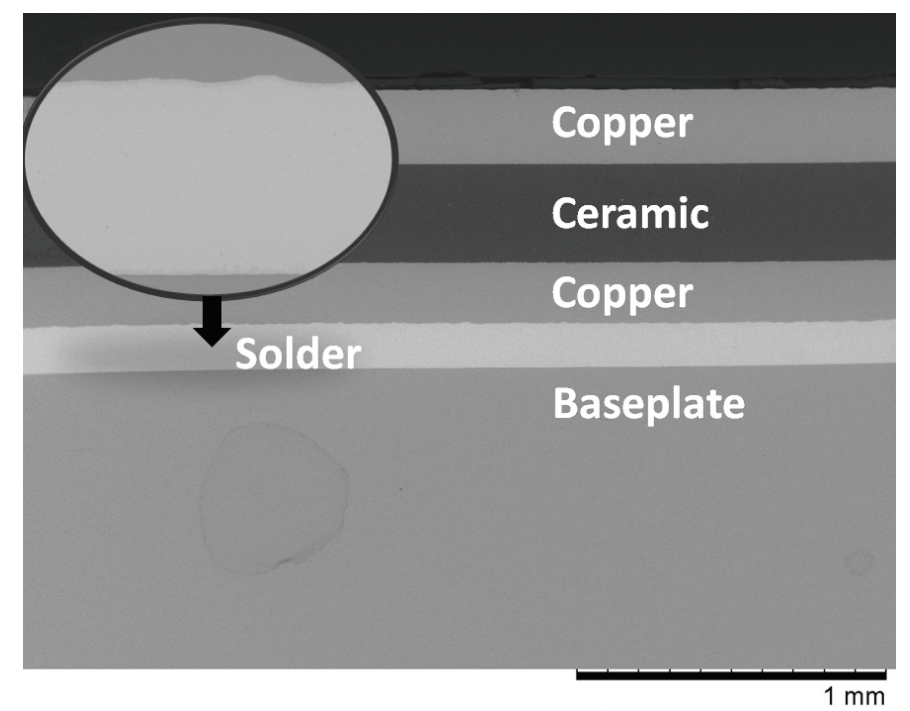

Figure 13: SEM cross-section scanning for adaptive cooling solution

In the case of adaptive cooling, shown in Fig. 13, due to the controller reduces the $\Delta \mathrm{T}$ so that the thermal-mechanical stress is reduced, there is no obvious void or crack is observed, and the solder layer thickness is kept as its original value. By comparing Fig. 12 and Fig. 13, it can be observed that the copper-ceramic interface in the substrate becomes more rough in fixed cooling than adaptive cooling. A compare of more zoomed-in scanning pictures on this interface are presented as in Fig. 14 and Fig. 15. 


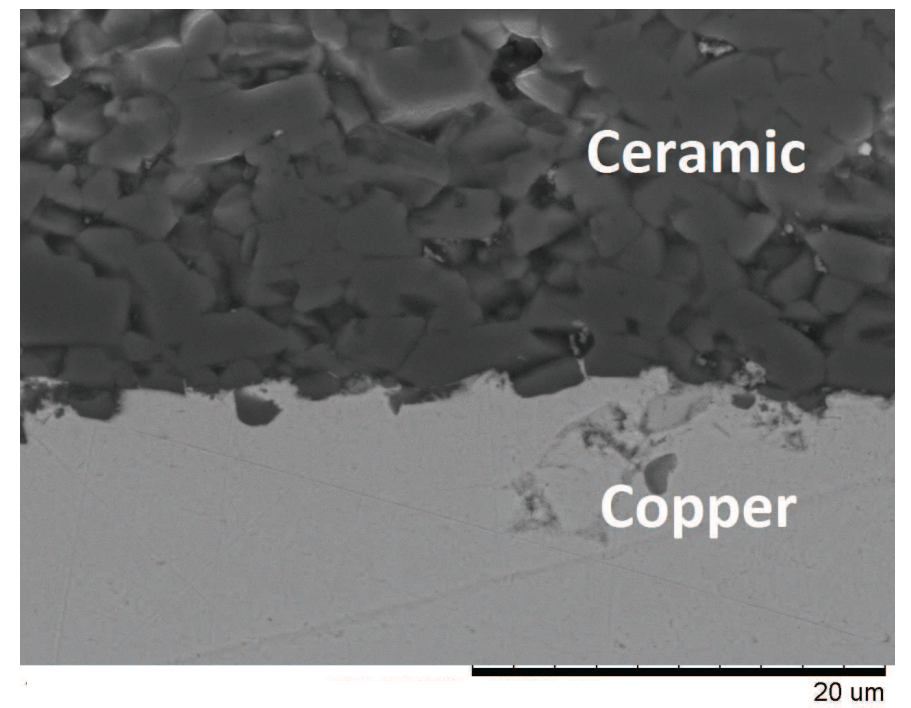

Figure 14: SEM cross-section scanning on substrate for fixed cooling solution

In Fig. 14, it can be seen that the thermal cycling test develops coarse ceramic grains in substrate when fixed cooling strategy is applied.

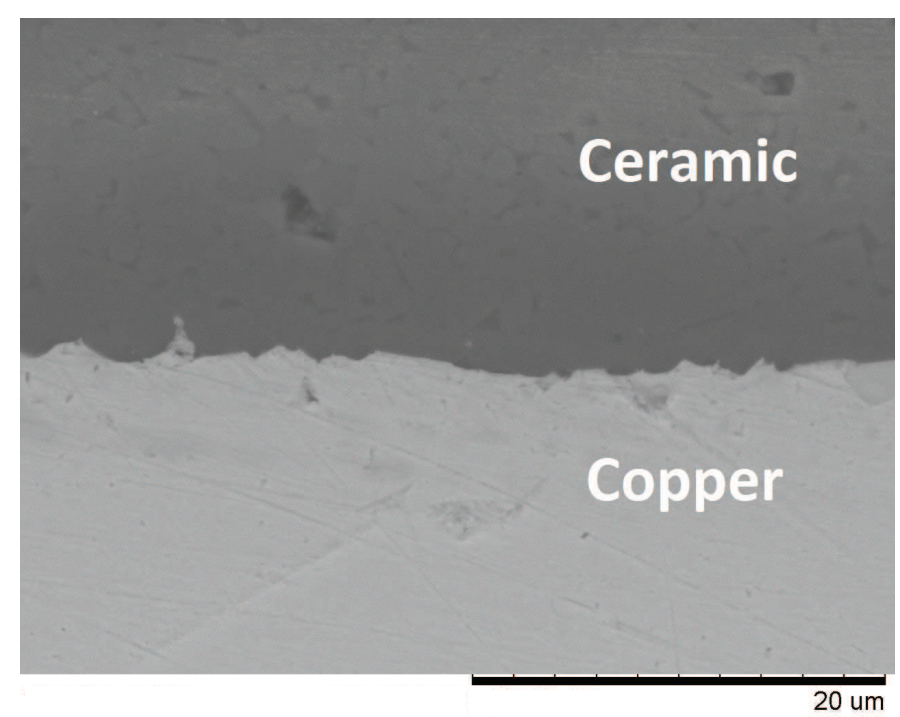

Figure 15: SEM cross-section scanning on substrate for adaptive cooling solution

While for adaptive cooling in Fig. 15, the ceramic grains in substrate 
are still smoothly arranged and the copper-ceramic interface is much more smooth than the fixed cooling one. Therefore, it can be summarised that the module with adaptive cooling solution has better performance in preventing voids generation and cracks propagation in solder layer, and even reduce copper-ceramic thermal-mechanical stress. The results clearly show that the proposed adaptive cooling can improve solder layer reliability and elongate device lifetime.

\section{Conclusion}

In this paper, an observer based dynamic adaptive cooling strategy has been proposed and validated. Considering the effect of temperature variation $\Delta T$ and average temperature $T_{m}$ on device reliability, the proposed cooling strategy uses multi-variable feedback control to regulate the device temperature against loads variations (e.g. power and ambient temperature change). With the developed feedback controller, a higher temperature reduction in $\Delta T$ is achieved than the increase in $T_{m}$. This regulated temperature result leads to an extended device lifetime. It can be observed from the experimental results that the proposed dynamic cooling strategy improves device lifetime beyond the existing fixed cooling method by adaptively adjust its cooling power to the loads. Due to the cooling power being controlled within its maximum value, the adaptive cooler does not require more power than the traditional fixed cooling.

Another original contribution of this work is the use of a temperature observer. Considering the difficulties in measuring temperatures at certain 
desired locations inside a commercialised module during operation, a temperature observer is proposed and validated. The proposed observer is able to estimate the internal module temperature (e.g. junction temperature or solder-layer temperature) in real-time without opening its package by just measuring the temperature at one accessible location (e.g. baseplate, heat sink). As the temperature dynamics information is reflected by the power dissipation and the ambient temperature signals, the observer doesn't rely on the dynamics of the temperature sensor. Therefore, the observer is especially suitable for power electronics temperature monitoring applications.

To verify the proposed cooling strategy, the observer and feedback controller were validated separately and then tested combined together on a commercial IGBT module (case-study). The test condition are scaled from domestic wind-turbine inverter applications, but the idea is very general and can be applied to a broad variety of power electronics applications (e.g. automotive, ship). During this case-study implementation, the power and temperature cycling were ran simultaneously for 3000 cycles. Temperature at the IGBT baseplate were compared and a reduction of $45^{\circ} \mathrm{C}$ in $\Delta T$ is achieved. The solder layer degradation were compared between the proposed adaptive cooling and the fixed cooling method by SAM and SEM scanning. The results demonstrate good result in improving device reliability and lifetime with the proposed adaptive cooling strategy. 


\section{References}

[1] M. Ciappa, Selected failure mechanisms of modern power modules, Microelectronics Reliability 42 (2002) $653-667$.

[2] T. Nakagawa, Replacement policies for a unit with random and wearout failures, Reliability, IEEE Transactions on R-29 (1980) 342 -344.

[3] D. Badenius, Random failure, Reliability, IEEE Transactions on R-19 (1970) $86-88$.

[4] X. Li, J. Qin, J. Bernstein, Compact modeling of mosfet wearout mechanisms for circuit-reliability simulation, Device and Materials Reliability, IEEE Transactions on 8 (2008) $98-121$.

[5] X. Wang, A. Castellazzi, P. Zanchetta, Regulated cooling for reduced thermal cycling of power devices, in: Power Electronics and Motion Control Conference (IPEMC), 2012 7th International, volume 1, pp. $238-244$.

[6] M. Bouarroudj, Z. Khatir, J. Ousten, F. Badel, L. Dupont, S. Lefebvre, Degradation behavior of 600v-200a igbt modules under power cycling and high temperature environment conditions, Microelectronics Reliability 47 (2007) 1719-1724.

[7] B. Ji, V. Pickert, B. Zahawi, M. Zhang, In-situ bond wire health monitoring circuit for igbt power modules, in: Power Electronics, Machines and Drives (PEMD 2012), 6th IET International Conference on, pp. 1 -6 . 
[8] V. Smet, F. Forest, J. Huselstein, F. Richardeau, Z. Khatir, S. Lefebvre, M. Berkani, Ageing and failure modes of igbt modules in hightemperature power cycling, Industrial Electronics, IEEE Transactions on 58 (2011) $4931-4941$.

[9] X. Perpina, J.-F. Serviere, J. Urresti-Ibanez, I. Cortes, X. Jorda, S. Hidalgo, J. Rebollo, M. Mermet-Guyennet, Analysis of clamped inductive turnoff failure in railway traction igbt power modules under overload conditions, Industrial Electronics, IEEE Transactions on 58 (2011) 2706 -2714 .

[10] G. Buiatti, J. Martin-Ramos, C. Garcia, A. Amaral, A. Cardoso, An online and noninvasive technique for the condition monitoring of capacitors in boost converters, Instrumentation and Measurement, IEEE Transactions on 59 (2010) $2134-2143$.

[11] L. Ngwendson, M. Sweet, E. Narayanan, An overview of the recent developments in high-voltage power semiconductor mos-controlled bipolar devices, in: Bipolar/BiCMOS Circuits and Technology Meeting, 2009. BCTM 2009. IEEE, pp. $198-205$.

[12] A. Morozumi, K. Yamada, T. Miyasaka, S. Sumi, Y. Seki, Reliability of power cycling for igbt power semiconductor modules, Industry Applications, IEEE Transactions on 39 (2003) 665 - 671.

[13] S. Igarashi, H. Kakiki, Y. Nishimura, T. Goto, Design of high reliability packaging for fuji high power module, in: Electrical Machines and Systems (ICEMS), 2011 International Conference on, pp. 1 -6. 
[14] U. Scheuermann, Reliability challenges of automotive power electronics, Microelectronics Reliability 49 (2009) 1319 - 1325.

[15] D. Murdock, J. Torres, J. Connors, R. Lorenz, Active thermal control of power electronic modules, Industry Applications, IEEE Transactions on 42 (2006) 552-558.

[16] Skuriat, Robert and Johnson, C. M., Direct substrate cooling of power electronics, Integrated Power Systems (CIPS), 2008 5th International Conference on (2008) $1-5$.

[17] U. Scheuermann, U. Hecht, Power cycling lifetime of advanced power modules for different temperature swings, PCIM Nuremberg (2002) 5964.

[18] Babcock, James Wittman and Tustaniwskyj, Jerry Ihor, Temperature control system for an electronic device in which device temperature is estimated from heater temperature and heat sink temperature, 1998. US Patent 5,844,208.

[19] W. J. Anderl, C. M. Huettner, Real time adaptive active fluid flow cooling, 2010. US Patent 7,733,649.

[20] D. K. Singh, F. I. Atallah, D. H. Allen, Fan speed control from adaptive voltage supply, 2013. US Patent 8,515,590.

[21] M. Musallam, C. M. Johnson, Real-time compact thermal models for health management of power electronics, Power Electronics, IEEE Transactions on 25 (2010) 1416-1425. 
[22] Schuh, William C, Smart temperature sensing device, 1999. US Patent $5,857,777$.

[23] X. Wang, A. Castellazzi, P. Zanchetta, Full-order observer based igbt temperature online estimation, in: Industrial Electronics Society, IECON 2014-40th Annual Conference of the IEEE, IEEE, pp. 14941498.

[24] C.-S. Yun, P. Regli, J. Waldmeyer, W. Fichtner, Static and dynamic thermal characteristics of igbt power modules, in: Power Semiconductor Devices and ICs, 1999. ISPSD'99. Proceedings., The 11th International Symposium on, IEEE, pp. 37-40.

[25] X. Wang, A. Castellazzi, P. Zanchetta, Observer based temperature control for reduced thermal cycling in power electronic cooling, Applied Thermal Engineering 64 (2014) 10-18.

[26] X. Wang, Y. Wang, A. Castellazzi, Reduced active and passive thermal cycling degradation by dynamic active cooling of power modules, in: Power Semiconductor Devices \& IC's (ISPSD), 2015 IEEE 27th International Symposium on, IEEE, pp. 309-312.

[27] Khapugin, AA and Martynenko, VA and Nischev, KN and Novopoltsev, MI, Calculation of stress-deformation states in power semiconductor modules with soldered interfaces (2013). 